\title{
Community phylogenetics require phylogenies reconstructed from plastid genomes
}

\author{
Lu Jin ${ }^{1}$, Jia-Jia Liu ${ }^{2}$, Tian-Wen Xiao ${ }^{2}$, Qiao-Ming Li ${ }^{3}$, Luxiang Lin ${ }^{3}$, Xiao-Na Shao ${ }^{3}$, \\ Chen-xin $\mathrm{Ma}^{2}$, Bu-Hang $\mathrm{Li}^{4}$, Xiang-Cheng $\mathrm{Mi}^{5}$, Xiu-Juan Qiao ${ }^{6}$, Ju-Yu Lian ${ }^{7}$, Gang Hao ${ }^{1}$, \\ and Xue-Jun Ge ${ }^{2}$ \\ ${ }^{1}$ College of Life Sciences, South China Agricultural University \\ ${ }^{2}$ Key Laboratory of Plant Resources Conservation and Sustainable Utilization, South \\ China Botanical Garden, Chinese Academy of Sciences \\ ${ }^{3}$ CAS Key Laboratory of Tropical Forest Ecology, Xishuangbanna Tropical Botanical \\ Garden, Chinese Academy of Sciences \\ ${ }^{4}$ School of Life Sciences, Sun Yat-Sen University \\ ${ }^{5}$ State Key Laboratory of Vegetation and Environmental Change, Institute of Botany, \\ Chinese Academy of Sciences \\ ${ }^{6}$ Key Laboratory of Aquatic Botany and Watershed Ecology, Wuhan Botanical Garden, \\ Chinese Academy of Sciences \\ ${ }^{7}$ Center of Plant Ecology, Core Botanical Gardens, Chinese Academy of Sciences
}

April 13, 2021

\begin{abstract}
Phylogenetic trees have been extensively used in community ecology. However, how the phylogenetic reconstruction affects ecological inferences is poorly understood. In this study, we reconstructed three different types of phylogenetic trees (a synthetictree generated using VPhylomaker, a barcode-tree generated using rbcL+matK+trnH-psbA and a genome-tree generated from plastid genomes) that represented an increasing level of phylogenetic resolution among 580 woody plant species from six dynamic plots in subtropical evergreen broadleaved forests of China. We then evaluated the performance of each phylogeny in estimations of community phylogenetic structure, turnover and phylogenetic signal in functional traits. As expected, the genome-tree was most resolved and most supported for relationships among species. For local phylogenetic structure, the three trees showed consistent results with Faith's PD and MPD; however, only the synthetic-tree produced significant clustering patterns using MNTD for some plots. For phylogenetic turnover, contrasting results between the molecular trees and the synthetic-tree occurred only with nearest neighbor distance. The barcode-tree agreed more with the genome-tree than the synthetic-tree for both phylogenetic structure and turnover. For functional traits, both the barcode-tree and genome-tree detected phylogenetic signal in maximum height, but only the genome-tree detected signal in leaf width. This is the first study that uses plastid genomes in large-scale community phylogenetics. Our results highlight the outperformance of genome-trees over barcode-trees and synthetic-trees for the analyses studied here. Our results also point to the possibility of Type I and II errors in estimation of phylogenetic structure and turnover and detection of phylogenetic signal when using synthetic-trees.
\end{abstract}

\section{Hosted file}

Msc_MER.pdf available at https://authorea.com/users/407630/articles/517902-communityphylogenetics-require-phylogenies-reconstructed-from-plastid-genomes 